\title{
Significance of initial ST segment changes for thrombolytic treatment in first inferior myocardial infarction
}

\author{
K Schröder, K Wegscheider, K-L Neuhaus, U Tebbe, R Schröder
}

\begin{abstract}
Objective-To evaluate the benefit to risk ratio of thrombolytic treatment in patients with small inferior acute myocardial infarction (AMI). Controlled studies relating the benefit from thrombolysis with initial electrocardiographic features are scarce and of limited sample size.

Design-Retrospective study of 728 patients with a first inferior AMI of six hours' duration from the Intravenous Streptokinase in Acute Myocardial Infarction (ISAM) study comparing streptokinase with placebo stratified by the initial sum ST segment elevation $(\Sigma S T)$ of $0.8 \mathrm{mV}$ or less and greater than $0.8 \mathrm{mV}$, and 636 patients from the International Joint Efficacy Comparison of Thrombolytics (INJECT) trial comparing double blind streptokinase with reteplase stratified by either $\Sigma S T$ or the presence of precordial ST segment depression.
\end{abstract}

Results-ISAM study patients with an $\Sigma S T$ of greater than $0.8 \mathrm{mV}$ had a significant mortality benefit from streptokinase throughout six years, while those with an $\Sigma S T$ of $0.8 \mathrm{mV}$ or less showed a trend to higher mortality at six months $(6 \cdot 3 \%$ streptokinase $v 5 \cdot 1 \%$ placebo). Despite significantly smaller infarcts and fewer clinical complications in patients with an $\Sigma S T$ of $0.8 \mathrm{mV}$ or less (ISAM and INJECT) or the absence of precordial ST segment depression (INJECT) thrombolytic treatment was associated with higher early mortality than in those with initially larger ST segment deviations.

Conclusion-Thrombolytic treatment in patients with inferior AMI presenting with larger ST segment deviations is associated with improved survival throughout six years. The risk to benefit ratio, however, in terms of early mortality in patients who have an $\Sigma S T$ of $0.8 \mathrm{mV}$ or less and no precordial ST segment depression may be unfavourable.

(Heart 1997;77:506-511)

Keywords: inferior myocardial infarction; ST segment deviation; thrombolytic treatment; mortality

Thrombolysis is claimed to be the treatment of choice for patients with acute myocardial infarction (AMI) of less than 12 hours dura- tion presenting with ST segment elevation on their admission electrocardiogram (ECG), irrespective of the site of infarction. ${ }^{12}$ Mortality benefit in patients with inferior AMI is less, however, than in those with anterior AMI, and there is conflicting evidence regarding the efficacy of reperfusion treatment in patients with small inferior AMI. ${ }^{3-6}$ Inclusion of these low risk patients in thrombolytic trials may be a major reason why the benefit of thrombolytic treatment in patients with inferior $\mathrm{AMI}$ has been less dramatic than in those with anterior AMI. ${ }^{5}$

Placebo controlled studies to clarify the precise indication for thrombolytic treatment in patients with inferior AMI are now unethical, and former controlled studies are scarce and limited by the small sample sizes. ${ }^{78}$ In the present study data from the Intravenous Streptokinase in Acute Myocardial Infarction (ISAM) study ${ }^{9}$ dataset, which provides a large well characterised cohort of patients, in whom initial ST segment elevation was measured, were reanalysed. Precordial ST segment depression was not measured in the ISAM study. Therefore, to bring the placebo controlled results on the initial sum ST segment elevation $(\Sigma S T)$ into relation with precordial ST segment depression, patients randomised in the recent International Joint Efficacy Comparison of Thrombolytics (INJECT) trial, were also reanalysed with stratification to both initial $\Sigma S T$ and presence of precordial ST segment depression. ${ }^{10}$

\section{Methods}

\section{PATIENTS}

The series comprised 728 patients from the ISAM study and 636 from the INJECT trial ECG substudy with a first inferior AMI. Patients older than 75 years in the ISAM study were excluded, while the INJECT trial was without an age limit. Each study was multicentre and double blind; the ISAM study compared streptokinase $1.5 \mathrm{MU}$ with placebo, and the INJECT trial compared reteplase, 10 + $10 \mathrm{MU}$ double bolus administration, with streptokinase. Patients presenting within six hours of the onset of symptoms in whom ST segment elevations of at least $0.1 \mathrm{mV}$ in two limb leads or $0.2 \mathrm{mV}$ in two contiguous chest leads were present were eligible.

\section{ELECTROCARDIOGRAPHIC ANALYSIS}

ST segment elevation was measured with lens intensified callipers to the nearest of $0.025 \mathrm{mV}$ 20 ms after the end of QRS complex with PR 
segment as the baseline. As in other studies ${ }^{78}$ $\Sigma$ ST was calculated from leads II, III, aVF, V5, and V6. In the INJECT trial substudy, ¿ST segment depression in leads V1-V4 was also measured. In accordance with other authors, ${ }^{5}$ significant precordial ST segment depression was defined as $0.1 \mathrm{mV}$ or more in at least two of leads V1-V4.

\section{ENZYME ANALYSIS}

In the ISAM study, serum activity of creatine kinase (CK) isoenzyme, $\mathrm{MB}$ fraction, was measured at two hour intervals. The enzyme infarct size was calculated from the area under the curve. In the INJECT trial, peak serum activity of creatine kinase isoenzyme was measured in the participating hospitals and is expressed as a fraction of the upper normal limit of the different methods used.

\section{QUANTITATIVE ANGIOGRAPHIC ANALYSIS}

In the ISAM study, cardiac catheterisation and angiography were performed one month after infarction. The ejection fraction was calculated from an angiogram in a $30^{\circ}$ right anterior oblique projection according to the area length method. Regional wall motion abnormalities were analysed in angiograms performed in the $30^{\circ}$ right anterior oblique and $60^{\circ}$ left anterior oblique projection, and calculated in square units as "regional dyssynergic area" (DA). Briefly, the end diastolic and end systolic silhouettes were superimposed and divided into 48 segmental radii. The area between radii shortening by less than two standard deviations (SD) and the 2 SD range for systolic shortening in healthy individuals were considered as the DA.

\section{STATISTICAL ANALYSIS}

Two sample comparisons were performed using the two sided Mann-Whitney $U$ test for continuous variables and Fisher's exact test for dichotomous variables. Survival curves were estimated according to Kaplan-Meier and compared using standardised log rank tests.

Two different methods were applied to estimate the dependence of six month mortality on increasing $\Sigma S T$ for different treatment groups. Cox regression analysis was used with the placebo or streptokinase treatment and $\Sigma$ ST elevation. This model assumes that the hazard of dying is proportional to the $\Sigma S T$ elevation in each treatment group, resulting in a global function of a type that is defined by the model. The significance of interaction terms-that is, the existence of different dependence functions in different treatment groups, can be tested within this model.

A moving average technique that is fairly common in time series analysis was used to detect deviations from the linear Cox model. First, discrete six month mortalities were calculated for intervals in steps of $0.1 \mathrm{mV}$. Second, weighted averages of the discrete mortalities were applied using a triangular weight function averaging over nine adjacent classes to achieve local smoothing of the crude mortality values. Ascending or descending trends in mortality were expressed by non-parametric rank biserial correlations $\tau_{\text {bis }}$ according to Glass, ${ }^{11}$ which can be tested with the Mann-Whitney U statistical test. The correlation coefficient and the corresponding $\mathrm{P}$ value can be interpreted in the same way as the Pearson correlation coefficient with $P$ value for continuous variables.

\section{Results}

CUT OFF POINT FOR LACK OF MORTALITY BENEFIT

Figure 1A (Cox regression analysis) and 1B (weighted averages) show six month mortality with the two applied estimation methods plotted by steps of increasing levels of $\Sigma S T$ on the baseline ECG for the ISAM patients (376 allocated to streptokinase and 352 to placebo) and INJECT trial patients with a first inferior AMI. In both models, patients in the ISAM study given placebo showed a continuous increase in mortality. In contrast, the curves for streptokinase treated patients were flat with some trend to lower mortality at higher $\Sigma S T$. The interaction term of the Cox model was significant $(2 \mathrm{P}=0.025$, fig $1 \mathrm{~A})$ as was the rank correlation $\tau_{\text {bis }}=0.23(P=0.029$ one sided, fig 1B) for the ISAM study placebo group, while the rank correlation in the ISAM streptokinase group was not significant $\left(\tau_{\text {bis }}=-0.02\right)$. In the INJECT trial, the mortality curves for patients randomised to thrombolytic treatment with either streptokinase or reteplase were also flat like those for the ISAM study streptokinase group (figs $1 \mathrm{~A}$ and $1 \mathrm{~B}$ ).
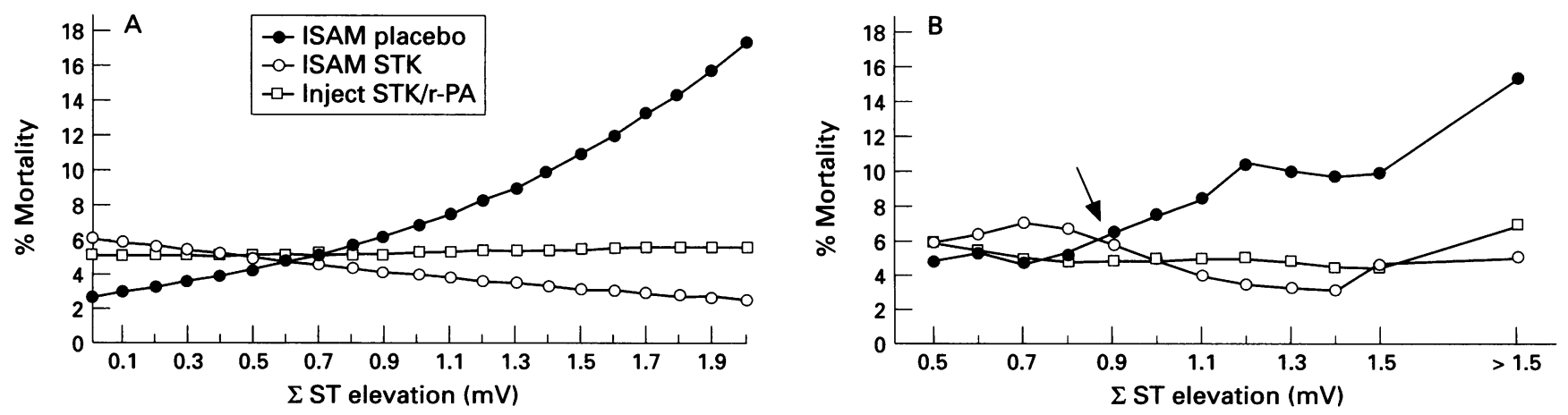

Figure 1 Plots showing six month mortality with increasing $\Sigma S T$ segment elevation on the baseline electrocardiogram in 352 patients allocated to placebo and 376 given streptokinase (STK) in the ISAM study by (A) Cox regression analysis and (B) the moving averages technique. (B) The mortality curves cross between 0.8 and $0.9 \mathrm{mV}$ (arrow). For comparison six month mortality for 636 patients of the INFECT trial who received thrombolytic treatment (reteplase ( $r-P A)$ or streptokinase) are displayed in each figure. 
Table 1 Enzymatic infarct sizes and left ventricular function according to an initial $\sum S T$ in patients with a first inferior AMI (ISAM study)

\begin{tabular}{|c|c|c|c|c|}
\hline & \multicolumn{2}{|c|}{$\sum S T \leqslant 0.8 \mathrm{mV}$} & \multicolumn{2}{|c|}{$\sum S T>0.8 \mathrm{mV}$} \\
\hline & $\begin{array}{l}\text { Streptokinase } \\
(n=180)\end{array}$ & $\begin{array}{l}\text { Placebo } \\
(n=174)\end{array}$ & $\begin{array}{l}\text { Streptokinase } \\
(n=132)\end{array}$ & $\begin{array}{l}\text { Placebo } \\
(n=92)\end{array}$ \\
\hline $\begin{array}{l}\text { CK-MB AUC }(\mathrm{IU} / \mathrm{ml} \times \mathrm{h}) \\
\text { Ejection fraction }(\%) \\
\text { DA-RAO (units) } \\
\text { DA-LAO (units) }\end{array}$ & $\begin{array}{l}1 \cdot 23(0 \cdot 9) \\
61 \cdot 2(12) \\
14 \cdot 8(14) \\
7 \cdot 5(9)\end{array}$ & $\begin{array}{l}1 \cdot 37(0 \cdot 9) \\
58 \cdot 7(12) \\
18 \cdot 3(15) \\
8 \cdot 6(11)\end{array}$ & $\begin{array}{l}1.98(1 \cdot 1)^{\star} \\
56.0(11) \ddagger \\
21.4(14) \dagger \\
8.7(9)\end{array}$ & $\begin{array}{r}2 \cdot 26(1 \cdot 3)^{\star} \\
51 \cdot 5(13)^{\star \#} \\
27 \cdot 0(16) \dagger \# \\
15 \cdot 3(10)^{\star} \delta\end{array}$ \\
\hline
\end{tabular}

Data are means (SD)

${ }^{\star} \mathrm{P}<0.0001 ;+\mathrm{P}<0.0005 ; \ddagger \mathrm{P}<0.005 v \sum \mathrm{ST} \leqslant 0.8 \mathrm{mV}$

$\S \mathrm{P}=0.0001 ; \# \mathrm{P}<0.05 v$ corresponding streptokinase groups.

AMI, acute myocardial infarction; CK-MB AUC, area under the creatine kinase isoenzyme, MB fraction, serum activity curve; DA, quantitative regional wall motion abnormality; RAO, right anterior oblique ventriculogram; LAO, left anterior oblique ventriculogram; $\Sigma S T$, sum of ST segment elevation on the baseline electrocardiogram from leads II, III, aVF, V5 and V6.

Table 2 Age and clinical event rates of 636 INFECT trial patients with a first inferior $A M I$ according to $S T$ segment deviations on the baseline electrocardiogram

\begin{tabular}{|c|c|c|c|c|c|c|}
\hline & $\begin{array}{l}\sum S T \leqslant 0 \cdot 8 \\
(n=336)\end{array}$ & $\begin{array}{l}\sum S T>0 \cdot 8 \\
(n=300)\end{array}$ & $P$ & $\begin{array}{l}S T \downarrow<0 \cdot 2 \\
(n=247)\end{array}$ & $\begin{array}{l}S T \downarrow \geqslant 0 \cdot 2 \\
(n=389)\end{array}$ & $P$ \\
\hline Age (years) & $60 \cdot 2$ & $61 \cdot 5$ & $0 \cdot 3$ & $59 \cdot 5$ & $61 \cdot 7$ & 0.05 \\
\hline Heart failure & $12 \cdot 8$ & $15 \cdot 7$ & $0 \cdot 3$ & $12 \cdot 6$ & $15 \cdot 2$ & $0 \cdot 4$ \\
\hline Cardiogenic shock & 3.9 & $6 \cdot 0$ & $0 \cdot 2$ & $4 \cdot 0$ & $5 \cdot 4$ & $0 \cdot 4$ \\
\hline AV block $2^{\circ}$ or $3^{\circ}$ & $9 \cdot 8$ & $13 \cdot 3$ & $0 \cdot 2$ & $7 \cdot 7$ & 13.9 & 0.02 \\
\hline Atrial fibrillation & $6 \cdot 0$ & $9 \cdot 7$ & 0.08 & $7 \cdot 3$ & $8 \cdot 0$ & $0 \cdot 8$ \\
\hline $\mathrm{VF} / \mathrm{VT}$ & $9 \cdot 5$ & $18 \cdot 0$ & 0.002 & $8 \cdot 1$ & $17 \cdot 0$ & 0.001 \\
\hline Recurrent MI & $5 \cdot 4$ & $5 \cdot 3$ & $1 \cdot 0$ & $5 \cdot 7$ & $5 \cdot 1$ & 0.7 \\
\hline Reischaemia & $9 \cdot 8$ & $14 \cdot 3$ & 0.08 & $10 \cdot 1$ & $13 \cdot 1$ & $0 \cdot 3$ \\
\hline PTCA/CABG & $22 \cdot 0$ & $25 \cdot 8$ & $0 \cdot 3$ & $21 \cdot 1$ & $25 \cdot 4$ & $0 \cdot 2$ \\
\hline
\end{tabular}

^Present after enrolment. Data (except age) are percentages.

PTCA/CABG, percutaneous transluminal coronary angioplasty/coronary artery bypass graft surgery; ST $\downarrow, \sum$ of precordial ST depression from leads V1-V4; VF/VT, ventricular fibrillation/sustained ventricular tachycardia; AV, atrioventricular; MI, myocardial infarction; $\sum S T$, sum of ST segment elevation on the baseline electrocardiogram from leads II, III, aVF, V5 and V6.

Basically, each approach gives the same impression. As the functional form of the global estimate is defined by the model rather than the data, however, Cox analysis seems to be less useful to define a cut off point. Therefore, the local smoothing method by moving average technique was used (fig 1B). According to this model mortality functions cross between $0.8 \mathrm{mV}$ and $0.9 \mathrm{mV}$. Thus, lack of mortality benefit from thrombolytic treatment was assumed for patients with a smaller $\Sigma S T$ on their baseline ECG defined by the cut off point of $\Sigma S T$ of $0.8 \mathrm{mV}$ or less.

\section{INFARCT SIZE AND LEFT VENTRICULAR}

FUNCTION

Patients with an $\Sigma S T$ of $0.8 \mathrm{mV}$ or less in the ISAM study had relatively low enzyme release and a relatively normal ejection fraction. Benefit from thrombolytic treatment was small and statistically non-significant. Patients in the streptokinase and placebo groups with an $\Sigma$ ST of more than $0.8 \mathrm{mV}$ had significantly larger enzymatic infarct sizes and worse left ventricular function (table 1). Streptokinase was associated with a significant improvement in global and regional ejection fraction compared with that from placebo. Regional wall motion of the left ventricle was mostly impaired with placebo and greatly improved by streptokinase when evaluated by left anterior oblique ventriculograms. This angiographic angulation assesses in particular inferoseptal and posterolateral segments. The difference in enzymatic infarct size was statistically significant in the 141 patients randomised within three hours from the onset of symptoms $(1.74(1.0) \mathrm{IU} / \mathrm{ml} \times$ hours streptokinase $v 2.33(1 \cdot 4)$ placebo, $\mathrm{P}=$ $0 \cdot 01)$.

MORTALITY IN PATIENTS OF THE ISAM STUDY Mortality at six months in patients with an $\Sigma S T$ of greater than $0.8 \mathrm{mV}$ allocated to streptokinase was $4.6 \%$ (seven of 152 patients died) compared with $10.4 \%$ in those given placebo (12 of 115 patients, $P=0.07$ ). The actuarial curves for survival in figure $2 \mathrm{~A}$ show a sustained mortality benefit throughout six years $(P=0.06)$. The cut off point of $0.8 \mathrm{mV}$ was derived from figure $1 \mathrm{~B}$. A shift to the right to $1.0 \mathrm{mV}$ was associated with mortality differences in favour of streptokinase, which were conventionally significant.

In contrast, an initial $\Sigma S T$ of $0.8 \mathrm{mV}$ or less in patients allocated to streptokinase was associated with a trend to higher six month mortality ( $6.3 v 5 \cdot 1 \%$ placebo) and no long term mortality differences between patients given streptokinase or matching placebo (fig 2B)

INJECT TRIAL: BASELINE CHARACTERISTICS AND CLINCIAL EVENT RATES ACCORDING TO INITIAL SEGMENT DEVIATIONS

Table 2 shows data according to $\Sigma S T$ and the absence or presence of significant precordial ST segment depression. In each group, stratified by larger ST segment deviations, patients
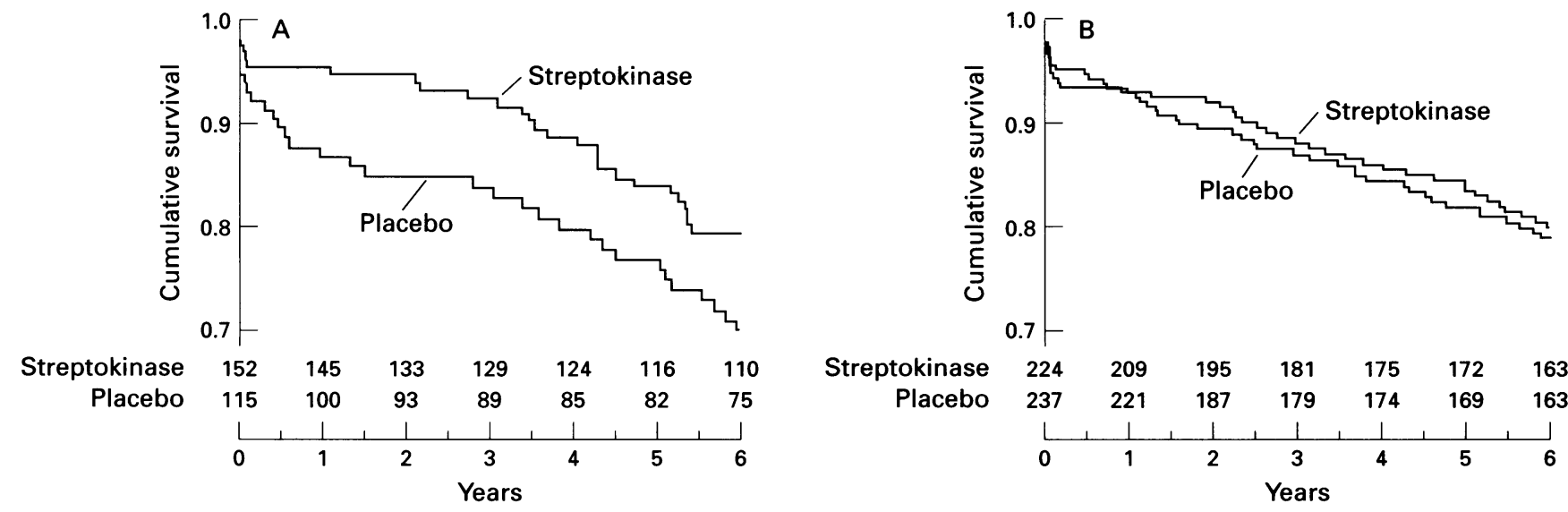

Figure 2 Kaplan-Meier survival curves for patients with a first inferior acute myocardial infarction; 376 allocated to streptokinase and 352 to placebo. The numbers of patients at risk at each time point are noted above the axes. (A) Patients with an $\Sigma S T$ of greater than $0.8 \mathrm{mV}$ on the baseline electrocardiogram; (B) those with an $\Sigma S T$ of $0.8 \mathrm{mV}$ or less. 
Table 3 Infarct sizes and six month mortality according to ST segment deviation on the baseline electrocardiogram (INFECT trial)

\begin{tabular}{lllllll}
\hline & $\sum S T \leqslant 0.8$ & $\sum S T>0.8$ & $P$ & $S T \downarrow<0.2$ & $S T \downarrow \geqslant 0.2$ & $P$ \\
\hline$\sum S T(\mathrm{mV})^{\star}$ & $0.52(0.2)$ & $1.32(0.5)$ & $<0.0001$ & $0.69(0.4)$ & $1.03(0.6)$ & $<0.0001$ \\
Peak CK $^{\star}$ & $8.3(6.3)$ & $12.8(8.6)$ & $<0.0001$ & $7.9(6.3)$ & $12.0(8.2)$ & $<0.0001$ \\
Mortality (\%) $\dagger$ & $6.0(20)$ & $5.3(16)$ & NS & $6.5(16)$ & $5.1(20)$ & NS \\
\hline
\end{tabular}

^Mean (SD).

†Values in parentheses are numbers of patients.

CK, creatine kinase isoenzyme serum activity; Peak, fraction of the upper normal limit; SST, sum of ST segment elevation on the baseline electrocardiogram from leads II, III, aVF, V5 and V6.
bating

were older and had a higher incidence of adverse cardiac events. The data in each group of either potentially smaller infarcts ( $\Sigma S T$ of $0.8 \mathrm{mV}$ and less or absence of precordial ST segment depression) or potentially larger infarcts ( $\Sigma S T$ of greater than $0.8 \mathrm{mV}$ or presence of precordial ST segment depression) are closely similar.

INJECT TRIAL: INFARCT SIZE AND MORTALITY ACCORDING TO ST SEGMENT DEVIATION

Table 3 shows that the ischaemic area at risk as measured from the extent of $\Sigma \mathrm{ST}$ was significantly smaller and the peak creatine kinase (CK) activity significantly lower in patients with either an $\Sigma S T$ of $0.8 \mathrm{mV}$ and less or absence of precordial ST segment depression than in those with larger ST segment deviation. In contrast, mortality at six months tended to be higher in patients with lesser ST segment deviation. The data are similar when analysed separately for patients randomised to reteplase or streptokinase.

Table 4 shows mortality and peak CK levels for patients with smaller or larger $\Sigma S T$ values combined with the presence or absence of precordial ST segment depression. The peak CK activity was smallest in patients with a smaller $\Sigma$ ST and absence of significant precordial ST segment depression, highest in patients with larger $\Sigma S T$ combined with precordial ST segment depression, and intermediate with either smaller $\Sigma S T$ and presence or larger $\Sigma S T$ and absence of precordial ST segment depression $(\mathrm{P}<0.0001)$

Paradoxically, as in ISAM study patients with smaller $\Sigma S T$ allocated to streptokinase, mortality tended to be higher in the group with a smaller $\Sigma S T$ and absence of precordial ST segment depression than in the other three groups with larger ST segment deviations in different combinations (mean mortality at six months $6.9 v 5.2 \%$ and mean peak CK $6.8 v$ $11 \cdot 8, \mathrm{P}<0.0001)$. Causes of death within the first 35 days in patients with a small $\Sigma S T$ and absence of precordial ST segment depression were recurrent myocardial infarction or ven-

Table 4 Mortality and peak creatine kinase values according to different combinations of $\sum S T$ and precordial ST segment depression (INFECT trial)

\begin{tabular}{|c|c|c|c|}
\hline \multirow[b]{2}{*}{$\sum$ of $S T$ deviation } & \multicolumn{2}{|l|}{ Mortality at } & \multirow{2}{*}{$\begin{array}{l}\text { Peak } \\
\text { creatine } \\
\text { kinase }\end{array}$} \\
\hline & 35 days & 6 months & \\
\hline $\begin{array}{l}\sum S T \leqslant 0.8 \text { and } S T \downarrow<0.2 \mathrm{mV} \\
\text { ST } \leqslant 0.8 \text { and } S T \downarrow \geqslant 0.2 \mathrm{mV} \\
\text { ST }>0.8 \text { and } S T \downarrow<0.2 \mathrm{mV} \\
\sum S T>0.8 \text { and } S T \downarrow \geqslant 0.2 \mathrm{mV}\end{array}$ & $\begin{array}{l}5 \cdot 1 \%(9 / 175) \\
3 \cdot 7 \%(6 / 161) \\
4 \cdot 2 \%(3 / 72) \\
4 \cdot 4 \%(10 / 228)\end{array}$ & $\begin{array}{l}6 \cdot 9 \%(12) \\
5 \cdot 0 \%(8) \\
5 \cdot 6 \%(4) \\
5 \cdot 3 \%(12)\end{array}$ & $\begin{array}{l}6 \cdot 8(5 \cdot 6)^{\star} \\
10 \cdot 0(6 \cdot 7) \\
10 \cdot 5(7 \cdot 1) \\
13 \cdot 5(8 \cdot 9)^{\star}\end{array}$ \\
\hline
\end{tabular}

Numbers in parentheses are numbers of patients except for peak CK (SD). $\Sigma S T$, sum of ST segment elevation on the baseline electrocardiogram from leads II, III, aVF, V5 and V6.

${ }^{\star} \mathrm{P}<0.0001 v$ the other three groups of ST segment deviations. tricular rupture in two patients each, and cardiac failure, asystole, sudden death, ventricular fibrillation during coronary angiography followed by cardiogenic shock, and cerebral haemorrhage once each.

\section{Discussion}

Despite more than a decade of extensive clinical trials the use of thrombolytic treatment in patients with inferior wall AMI continues to be controversial..$^{1-612}$ Some investigators have recommended that thrombolysis should be limited to patients with anterior AMI. ${ }^{312}$ Others have claimed that thrombolytic treatment should be given to all patients presenting with ST segment elevation. Although the results are less convincing in patients with inferior AMI, treatment worldwide may save tens of thousands of lives each year. ${ }^{12}$ Only two controlled studies have attempted to relate the benefit from thrombolysis for first inferior AMI to the amount of myocardium at risk as assessed by the initial $\Sigma S T$ or the presence of significant precordial ST segment depression, respectively. ${ }^{78}$ Mortality risk could not be assessed accurately, however, because of the sample size and very low in-hospital mortality. The GISSI trial ${ }^{13}$ related hospital mortality to the number of leads with ST segment elevation on the admission ECG. Mortality benefit from streptokinase administration increased progressively with the number of affected ECG leads. Unfortunately, data are not provided for the subgroup of patients with inferior AMI.

PREDICTION OF INFARCT SIZE

The value of ST segment elevation on the admission ECG in patients with AMI as a prognostic indicator has been established over many years. ${ }^{14-16}$ The present ISAM study derived analysis showed significantly larger enzymatic infarct size and worse left ventricular function in patients with inferior AMI who had an $\Sigma S T$ of more than $0.8 \mathrm{mV}$ than those with an $\Sigma S T$ of $0.8 \mathrm{mV}$ and less receiving placebo or streptokinase (table 1).

Currently, the presence or absence of precordial ST segment depression is preferred for prediction of infarct size in inferior AMI. ${ }^{568}$ Precordial ST segment depression reflects a larger area of ischaemia with extension to posterolateral or inferoseptal regions, indicative of larger enzymatic infarct size and worse regional and global left ventricular function. ${ }^{17-19}$ Studies before the thrombolytic era showed that these patients had higher short and long term mortality. ${ }^{517} 18$ Bates et $a l^{5}$ also reported higher complication rates and worse left ventricular function after reperfusion treat- 
ment. These findings are confirmed by the present study, which, in addition, shows that the data are closely similar in patients stratified by an $\Sigma S T$ of greater than $0.8 \mathrm{mV}$ (tables 2 and 3). This suggests that large inferior ST segment elevation often coincides with precordial ST segment depression, and that for simplicity the presence of precordial ST segment depression may be sufficient to identify patients with potentially larger infarcts. Several factors, however, alter the magnitude and direction of the ST segment vector in AMI. ${ }^{17}$ Concomitant right ventricular involvement may attenuate precordial ST segment depression and lateral involvement may augment it..$^{20}$ In our INJECT trial derived analysis the magnitude of inferior or inferolateral ST segment elevation correlated only marginally with precordial ST segment depression ( $r=$ 0.50 and 0.44 , respectively). Therefore, and as data displayed in table 4 clearly show, definition of potentially small inferior AMI must include the absence of precordial ST segment depression and a smaller sum of inferior ST segment elevation.

\section{BENEFIT OF THROMBOLYTIC TREATMENT ON INFARCT SIZE}

Streptokinase in patients with inferior AMI and an initial $\Sigma S T$ of greater than $0.8 \mathrm{mV}$ significantly improved enzymatic infarct size and left ventricular function (table 1). This result corroborates previous studies in which significant benefit from thrombolysis in patients with either a larger $\Sigma S T$ or the presence of significant precordial ST segment depression was described. ${ }^{78}$ In keeping with the present analysis in patients with lesser amounts of ST segment deviation, left ventricular function in the control groups was relatively well preserved and improvement by thrombolytic treatment was small. ${ }^{78}$

These data are valid for patient groups of sufficient sample size. In individual patients, however, the prognostic merit of the extent of initial ST segment deviation is more limited. The overall correlations with enzymatic infarct size or final left ventricular function are fairly low. ${ }^{81516}$ In fact, one of three patients with inferior AMI, who had initially smaller ST segment elevations, developed a larger infarct. ${ }^{16}$ Nevertheless, thrombolytic treatment in this generally low risk population would not be justified if an adverse effect on early mortality is suspected.

\section{EVIDENCE OF INCREASED MORTALITY RISK WITH THROMBOLYTIC TREATMENT?}

In the INJECT trial derived analysis mortality closely parallels that of the streptokinase treated patients in the ISAM study (figs 1A and $1 \mathrm{~B})$. Streptokinase clearly improved short and long term survival in patients who had an $\Sigma S T$ of more than $0.8 \mathrm{mV}$ compared with that in those who received placebo in the ISAM study (figs 1 and 2). The magnitude of mortality benefit approaches that in patients with anterior AMI.

In patients with a smaller $\Sigma S T$, however, the death rates at six months tended to be higher in those allocated to streptokinase than in those allocated to placebo. In the INJECT trial all patients had thrombolytic treatment. Patients with lesser initial ST segment changes had significantly smaller infarcts and fewer clinical complications than those with an $\Sigma S T$ of greater than $0.8 \mathrm{mV}$ or the presence of precordial ST segment depression (tables 2 and 3). In contrast, as in the streptokinase group of the ISAM study (fig 1), early mortality was higher in patients who had lesser ST segment deviations (tables 3 and 4). The virtually identical findings with thrombolytic treatment in both trials not only support the assumption of no benefit but even provide suggestive evidence for a slight increase in early mortality risk in patients with potentially small inferior AMI. As the sample size is far too small, an increased mortality risk cannot be substantiated by our data. It becomes apparent, however, if we assume that a small improvement in left ventricular performance within the near normal range (table 1 ) is not associated with any mortality benefit. Then the risks inherent to thrombolytic treatment will not be counterbalanced. Thrombolysis carries the risk of lethal bleeding complications and is associated with an early mortality hazard supposedly caused by reperfusion injury. ${ }^{1}$ The Fibrinolytic Therapy Trialists' Collaborative Group ${ }^{1}$ overview showed a non-significant overall mortality benefit from thrombolytic treatment of only $0.9 \%$ (absolute $7.5 v 8.4 \%$ control) for patients with inferior ST segment elevation, and there was an excess of deaths soon after thrombolysis $(2.6 \% \quad v \quad 1.9 \%$ allocated control). ${ }^{1}$ As an excess of early deaths was not observed in patients with anterior ST segment elevation and the mortality benefit of streptokinase in patients presenting with larger inferior ST segment elevation was comparable to that known for anterior AMI patients (fig 1), the excess of early deaths of patients with inferior AMI are probably mainly caused by those presenting with small ST segment deviations, which are not outweighed by later mortality benefit.

\section{LIMITATIONS}

In the initial ISAM study analysis the amount of ST segment elevation on the admission ECG was not a prognostic indicator for an early mortality risk in patients with inferior AMI allocated streptokinase (in contrast to placebo). This might have reflected a game of chance. Confirmation by the INJECT trial ECG substudy, however, makes a play of chance unlikely. The present evaluation is clearly a retrospective subgroup analysis with its limitations. Such analyses are usually appropriate to generate a hypothesis for testing in a prospective randomised controlled trial. It is unlikely, however, that such an effort will be undertaken in the future because of the huge sample size required to prove a small mortality difference in patients, who generally have a relatively low risk of dying. Thus, the suspicion of a small increase in early mortality caused by thrombolytic treatment in patients with a small inferior AMI will probably not be 
substantiated on the basis of a controlled trial

How should the emerging concern about a possibly harmful effect of an accepted treatment in patients with AMI be managed? Such potentially important clinical data should not be ignored but rather presented to the medical community. Physicians may then decide how to consider these effects when making clinical decisions concerning patients with AMI.

\section{Conclusions}

Thrombolytic treatment is clearly indicated in patients with inferior AMI presenting within six hours from the onset of symptoms with either large infero(lateral) ST segment elevation or concomitant precordial ST segment depression. In addition, thrombolysis is beneficial in patients with an ST segment elevation of $0.1 \mathrm{mV}$ or more in lead $\mathrm{V} 4 \mathrm{R}$, indicating right ventricular involvement. ${ }^{21}$ In contrast, the benefit from such treatment in patients with an initial $\Sigma S T$ of $0.8 \mathrm{mV}$ or less combined with a precordial $\Sigma S T$ segment depression of less than $0.2 \mathrm{mV}$ is at least small. Thrombolysis may even carry an unfavourable benefit to risk ratio, particularly in patients at high risk for intracranial haemorrhage such as the elderly and those with hypertension or previous cerebrovascular events. ${ }^{22} 23$ Thrombolytic treatment should be considered only if small ST segment deviations are associated with advanced heart block or haemodynamic instability indicating larger infarcts. ${ }^{6}$ As with all treatments, patient selection has to be guided by weighing the potential benefit against the potential risks. In the INJECT trial ECG substudy, $28 \%$ of patients with inferior AMI had small ST segment deviations (table 4). For inclusion into the INJECT trial ST segment elevation of $0.1 \mathrm{mV}$ or greater in at least two limb leads was required. As it is common clinical practice to treat those with even lower ST segment elevations with thrombolytics, the proportion of patients in whom thrombolysis should be avoided is probably even larger. Whether such patients may benefit from direct coronary angioplasty is not known and remains speculative. The prognosis for patients with a first small inferior AMI is usually good and so any aggressive therapeutic approach may cause more harm than benefit.

1 Fibrinolytic Therapy Trialists' (FTT) Collaborative Group. Indications for fibrinolytic therapy in suspected acute myocardial infarction: collaborative overview of domised trials of more than 1000 patients. Lancet 1994; 343:311-22.

2 ISIS-2 (Second International Study of Infarct Survival) Collaborative Group. Randomised trial of intravenous streptokinase, oral aspirin, both, or neither among 17187 cases of suspected acute myocardial infarction: ISIS-2. Lancet 1988;ii:349-60.

3 Kennedy JW, Atkins JM, Goldstein S, Jaffe AS, Lamberew $\mathrm{CT}$, McIntyre KM, et al. Recent changes in managemen of acute myocardial infarction: implication for emergency care physicians. F Am Coll Cardiol 1988;11:446-9.

4 Braunwald $\mathrm{E}$. Thrombolytic reperfusion of acute myocardial infarction: resolved and unresolved issues. $f \mathrm{Am} \mathrm{Col}$ Cardiol 1988;12:85-92A

5 Bates ER, Clemmensen PM, Califf RM, Gorman LE, Aronson LG, George BS, et al. Precordial ST segment depression predicts a worse prognosis in inferior infarction despite reperfusion therapy. $7 \mathrm{Am}$ Coll Cardiol 1990 ; 16:1538-44.

6 Murphy JF, Jain NL, Kahn MG, Romero CA. Inferior wall myocardial infarction and thrombolytic therapy: an appraisal of clinical heterogeneity and indications for treatment [abstract]. $\mathcal{F}$ Am Coll Cardiol 1995;25:2C7.

7 Bar FW, Vermeer F, De Zwaan C, Ramentol M, Braat S Simoons ML, et al. Value of admission electrocardiogram in predicting outcome of thrombolytic therapy in acute myocardial infarction. Am $\mathcal{f}$ Cardiol 1987;59:6-13.

8 Willems IL, Willems RJ, Willems GM, Arnold AER, Van de Werf F, Verstraete M. Significance of initial ST segment elevation and depression for the management of thrombolytic therapy in acute myocardial infarction. Circulation 1990;82:1147-58.

9 ISAM (Intravenous Streptokinase in Acute Myocardial Infarction) Study Group. A prospective trial of intravenous streptokinase in acute myocardial infarction (ISAM): mortality, morbidity, and infarct size at 21 days. $N$ Engl $\mathcal{F}$ Med 1986;314:1465-71.

10 Schröder R, Wegscheider K, Schröder K, Dissmann R, Meyer-Sabellek W, for the INJECT Trial Group. Extent of early ST segment elevation resolution: a strong predictor of outcome in patients with acute myocardial predictor of outcome in patients with acute myocardial thrombolytic regimes. $\mathcal{f} \mathrm{Am}$ Coll Cardiol 1995;26: 1657-64.

11 Glass GV. Note on rank-biserial correlation. Educational and Psychological Measurement 1966;26:623-31.

12 Tobé TJM. Is thrombolytic therapy in acute inferior myocardial infarction really better than conventional treatcardial infarction really better than

13 Mauri F, Gasparini M, Barbonaglia L, Santoro E, Franzosi MG, Tognoni G, et al. Prognostic significance of the extent of myocardial injury in acute myocardial infarction treated by streptokinase (the GISSI Trial). $A m \mathcal{F}$ Cardiol 1989;63:1291-5.

14 Muller JE, Maroko PR, Braunwald E. Precordial electrocardiographic mapping: a technique to assess the efficacy of interventions designed to limit infarct size. Circulation 1978;57:1-18.

15 Aldrich HR, Wagner NB, Boswick J, Corsa AT, Jones MG Grande $\mathrm{P}$, et al. Use of initial ST-segment deviation for prediction of final electrocardiographic size of acute myocardial infarcts. Am $₹$ Cardiol 1988;61:749-53.

16 Schröder $R$ Linderer $T$, Brüggemann $T$, Neuhaus $K-I$ Tebbe U, Wegscheider $K$, for the ISAM Study Group. Rationale for thrombolysis later than 4-6 h from sympRationale for thrombolysis later than 4-6 h from symptom onset, and in patients with smaller my.

17 Mirvis DM. Physiologic bases for anterior ST segment depression in patients with acute inferior wall myocardial depression in patients with acute inferior wall
infarction. Am Heart $\mathcal{F} 1988 ; 116: 1308-22$.

18 Hlatky MA, Califf RM, Lee KL, Pryor DB, Wagner GS Rosati RA. Prognostic significance of precordial ST-segment depression during inferior acute myocardial infarction. Am ₹ Cardiol 1985;55:325-9.

19 Wong CK, Freedman SB. Implications of ST changes in reperfusion management of acute myocardial infarction Eur Heart $\mathcal{f} 1994 ; 15: 1385-90$

20 Lew AS, Maddahi J, Shah PK, Weiss AT, Peter T, Berman DS, et al. Factors that determine the direction and magnitude of precordial ST-segment deviations during inferior wall acute myocardial infarction. $A m \mathcal{F}$ Cardio 1985;55:883-8.

21 Zehender M, Kasper W, Kauder E, Geibel A, Schönthaler $\mathrm{M}$, Olschewski $\mathrm{M}$, et al. Eligibility for and benefit of thrombolytic therapy in inferior myocardial infarction: focus on the prognostic importance of right ventricular infarction. $\mathcal{F}$ Am Coll Cardiol 1994;24:362-9.

22 Simoons ML, Maggioni AP, Knatterud G, Leimberger JD, de Jaegere $\mathrm{P}$, van Domburg $\mathrm{R}$, et al. Individual risk assessment for intracranial haemorrhage during thrombolytic therapy. Lancet 1993;342:1523-8.

23 Gore JM, Granger CB, Simoons ML, Sloan MA, Weaver WD, White HD, et al. Stroke after thrombolysis. Mortality and functional outcomes in the GUSTO-I Trial. Circulation 1995;92:281-8. 